2016-06-22

\title{
Diverse impacts of the rs58542926 E167K variant in TM6SF2 on viral and metabolic liver disease phenotypes
}

\section{Eslam, M}

http://hdl.handle.net/10026.1/5267

\subsection{2/hep.28475}

Hepatology

Ovid Technologies (Wolters Kluwer Health)

All content in PEARL is protected by copyright law. Author manuscripts are made available in accordance with publisher policies. Please cite only the published version using the details provided on the item record or document. In the absence of an open licence (e.g. Creative Commons), permissions for further reuse of content should be sought from the publisher or author. 
Diverse impacts of the rs58542926 E167K variant in TM6SF2 on viral and metabolic liver disease phenotypes

\author{
Mohammed Eslam ${ }^{1}$, Alessandra Mangia ${ }^{2}$, Thomas Berg ${ }^{3}$, Henry Lik Yuen Chan ${ }^{4}$, William L. \\ Irving ${ }^{5}$, Gregory J. Dore ${ }^{6,7}$, Maria Lorena Abate ${ }^{8}$, Elisabetta Bugianesi ${ }^{8}$, Leon A. Adams ${ }^{9}$, \\ Mustafa AM Najim ${ }^{1,10}$, Luca Miele ${ }^{11}$, Martin Weltman ${ }^{12}$, Lindsay Mollison ${ }^{13}$, Wendy Cheng ${ }^{14}$, \\ Stephen Riordan ${ }^{15}$, Janett Fischer ${ }^{3}$, Manuel Romero-Gomez ${ }^{16}$, Ulrich Spengler ${ }^{17}$, Jacob \\ Nattermann $^{17}$, Antony Rahme ${ }^{1}$, David Sheridan ${ }^{18}$, David R. Booth ${ }^{19}$, Duncan McLeod ${ }^{20}$, Elizabeth \\ Powell $^{21}$, Christopher Liddle ${ }^{1}$, Mark W. Douglas ${ }^{1,22}$, David van der Poorten ${ }^{1}$ and Jacob George ${ }^{1}$ \\ for the International Liver Disease Genetics Consortium (ILDGC).
}

${ }^{1}$ Storr Liver Centre, Westmead Millennium Institute and Westmead Hospital, University of Sydney, NSW, Australia

${ }^{2}$ Division of Hepatology, Ospedale Casa Sollievo della Sofferenza, IRCCS, San Giovanni Rotondo, Italy

${ }^{3}$ Section of Hepatology, Clinic for Gastroenterology and Rheumatology, University Clinic Leipzig, Leipzig, Germany

${ }^{4}$ Department of Medicine and Therapeutics, Prince of Wales Hospital, The Chinese University of Hong Kong, Hong Kong, China

${ }^{5}$ NIHR Biomedical Research Unit in Gastroenterology and the Liver, University of Nottingham, Nottingham, United Kingdom,

${ }^{6}$ Kirby Institute, The University of New South Wales, Sydney, NSW, Australia.

${ }^{7}$ St Vincent's Hospital, Sydney, NSW, Australia.

${ }^{8}$ Division of Gastroenterology and Hepatology, Department of Medical Science, University of Turin, Turin, Italy

${ }^{9}$ School of Medicine and Pharmacology, Sir Charles Gairdner Hospital Unit, University of Western Australia, Nedlands, WA, Australia

${ }^{10}$ Department of Medical Laboratories Technology, Faculty of Applied Medical Sciences, Taibah University, Medina, Saudi Arabia

${ }^{11}$ Department of Internal Medicine, Catholic University of the Sacred Heart, Rome, Italy

${ }^{12}$ Department of Gastroenterology and Hepatology, Nepean Hospital, Sydney, NSW, Australia 
${ }^{13}$ Department of Gastroenterology and Hepatology, Fremantle Hospital, Fremantle, WA, Australia

${ }^{14}$ Department of Gastroenterology \& Hepatology, Royal Perth Hospital, WA, Australia.

${ }^{15}$ Gastrointestinal and Liver Unit, Prince of Wales Hospital and University of New South Wales, Sydney, NSW, Australia

${ }^{16}$ Unit for The Clinical Management of Digestive Diseases and CIBERehd, Hospital Universitario de Valme, Sevilla, Spain.

${ }^{17}$ Department of Internal Medicine I, University of Bonn, Bonn, Germany

${ }^{18}$ Institute of Translational and Stratified Medicine, Plymouth University, United Kingdom

${ }^{19}$ Institute of Immunology and Allergy Research, Westmead Hospital and Westmead Millennium Institute, University of Sydney, NSW, Australia.

${ }^{20}$ Department of Anatomical Pathology, Institute of Clinical Pathology and Medical Research (ICPMR), Westmead Hospital, Sydney, Australia

${ }^{21}$ The University of Queensland, School of Medicine, Princess Alexandra Hospital, Woolloongabba, QLD, Australia

${ }^{22}$ Centre for Infectious Diseases and Microbiology, Marie Bashir Institute for Infectious Diseases and Biosecurity, University of Sydney at Westmead Hospital, Westmead NSW, Australia 


\section{Corresponding Author}

Jacob George

Department of Medicine

Westmead Hospital

Westmead, NSW 2145

Ph: 61-2-98457705; Fx 61-2-96357582

Email: jacob.george@sydney.edu.au

Running title: $T M 6 S F 2$ and viral hepatitis

Key words: Chronic hepatitis C, Chronic hepatitis B, non-alcoholic steatohepatitis, viral load, TM6SF2, fibrosis, Steatosis, metabolic.

Competing Financial Interests: The authors declare no competing financial interests.

Acknowledgements: We would like to thank all the patients for their participation in this study. ME, MD and JG are supported by the Robert W. Storr Bequest to the Sydney Medical Foundation, University of Sydney; a National Health and Medical Research Council of Australia (NHMRC) Program Grant (1053206) and Project grants 1006759 and 1047417. GD is supported by an NHMRC Fellowship (1028432). ME is supported by an International Postgraduate Research Scholarships (IPRS) and an Australian Postgraduate Award (APA) of the University of Sydney.

Author Contributions: M.E., J.G. conceived the research. Enrolling of patients, clinical phenotype, data collation and sample acquisition/DNA preparation was performed by M.E, J.G., C.L., D.B, M.W.D, M.R-G., T.B, G.J.D., H.L.Y.C, W.L.I, D.S, M.L.A., L.A.A., A.M., M.W., E.B., U.S., O.S., J.F., L.M., W.C., E.P., J.N. and S.R. Genotyping was performed by A.R and R.W. Histological analysis of tissues and scoring was conducted by D.M. Statistical analysis and interpretation of results was performed by M.E and J.G. The manuscript was principally written and revised by M.E and J.G. All authors critically reviewed the manuscript for important intellectual content and approved the final submitted manuscript. 


\section{Abstract (N=264 words)}

A genome-wide exome association study has identified the transmembrane 6 superfamily member 2 (TM6SF2) rs58542926 variant encoding an E167K substitution as a genetic determinant of hepatic steatosis in nonalcoholic fatty liver disease (NAFLD). The role of this variant across a spectrum of liver diseases and pathologies and on serum lipids comparing viral hepatitis to NAFLD, and viral load in chronic viral hepatitis, and its intrahepatic molecular signature, have not been well characterized. We undertook detailed analyses in 3,260 subjects with viral and non-viral liver diseases and in healthy controls. Serum inflammatory markers and hepatic expression of TM6SF2 and genes regulating lipid metabolism were assessed in a subset with chronic hepatitis C (CHC). The rs58542926 T allele was more prevalent in 502 NAFLD patients than controls $(\mathrm{p}=0.02)$, but not different in cohorts with $\mathrm{CHC}(\mathrm{n}=2,023)$ and $\mathrm{CHB}(\mathrm{n}=$ 507). The $\mathrm{T}$ allele was associated with alterations in serum lipids and hepatic steatosis in all diseases, and with reduced hepatic TM6SF2 and MTTP expression expression. Interestingly, the substitution was associated with reduced $\mathrm{CHC}$ viral load, but increased HBV-DNA. The rs58542926 $\mathrm{T}$ allele had no effect on inflammation, impacted on $\geq \mathrm{F} 2$ fibrosis in $\mathrm{CHC}$ and NAFLD assessed cross-sectionally (OR: 1.39 (95\% CI = 1.04-1.87 and OR: $1.62,95 \%$ CI: 1.03 2.52, respectively, $\mathrm{p}<0.03$ for both), but had no effect on fibrosis progression in 1174 patients with CHC and a known duration of infection. We conclude that the TM6SF2 E167K substitution promotes steatosis and lipid abnormalities in part by altering TM6SF2 and MTTP expression and differentially impacts $\mathrm{CHC}$ and $\mathrm{CHB}$ viral load, while effects on fibrosis are marginal. 


\section{INTRODUCTION}

Ectopic lipid accumulation associated with the increasing worldwide prevalence of obesity $\left({ }^{1}\right)$ is linked with tissue meta-inflammation $\left({ }^{2}\right)$, resulting in organ dysfunction, excess morbidity and mortality $\left({ }^{3}\right)$. In this context, hepatic rather than visceral fat deposition is more tightly linked with the metabolic complications of obesity $\left({ }^{4}\right)$ and it is therefore not surprising that steatosis is now the most commonly observed histological abnormality across a spectrum of liver diseases $\left(^{5}\right)$. The heritability of steatosis is therefore of considerable interest, with a landmark genome-wide association study (GWAS) $\left({ }^{6}\right)$ and subsequent reports $\left(^{7}\right)$ demonstrating unequivocal evidence that a non-synonymous single-nucleotide polymorphism (SNP) in patatinlike phospholipase domain containing 3 (PNPLA3) rs738409 is associated not only with steatosis severity, but with the extent of fibrosis in NAFLD $\left({ }^{8}\right)$. More recently, in 7176 individuals of European ancestry, a GWAS meta-analysis in NAFLD using 2.4 million SNPs imputed to HapMap, identified additional common variants at 5 loci containing multiple genes (NCAN/TM6SF2/CILP2/PBX4) that impacts steatosis $\left(^{7}\right)$. A subsequent genome-wide exome association study with functional analysis $\left({ }^{9}\right)$ identified an $r s 58542926 C>T$ variant in the transmembrane 6 superfamily member $2(T M 6 S F 2)$ gene, which encodes an amino acidic substitution $(\mathrm{E} 167 \mathrm{~K})$ as the causative variant for the GWAS signal. The impact of TM6SF2 $r s 58542926$ as a determinant of liver fat in NAFLD is well-established $\left({ }^{10,11,12}\right)$. While there is evidence suggesting a role for this variant in NAFLD-associated liver fibrosis, this finding is not unequivocal $\left({ }^{10,11,12,13,14}\right)$, and is debatable in the case of CHC $\left({ }^{15,16}\right)$. There is no data on the role of $r s 58542926$ in chronic hepatitis $\mathrm{B}$ (CHB).

With regard to the metabolic consequences of harboring the $r s 58542926 \mathrm{~T}$ allele, an association with lower levels of serum total cholesterol, LDL-cholesterol (LDL-C) and triglycerides (TG), and protection from atherosclerosis and cardiovascular events in NAFLD 
$(9,11,17)$ has been reported. This cardiovascular risk mitigation is likely a consequence of data (from mouse models and cell lines), suggesting that TM6SF2 modulates hepatic lipoprotein export $\left({ }^{9,18}\right)$. However, corroborating human data is absent, but required, given the limitations of the cell lines used in published reports $\left({ }^{15}\right)$. VLDL secretion is nearly absent in Huh7 cells $\left({ }^{19}\right)$ and HepG2 cells secrete relatively dense, lipid poor apolipoprotein B (apoB)-containing particles, unlike the buoyant VLDL particles secreted in vivo $\left({ }^{20}\right)$. The impact of TM6SF2 rs58542926 polymorphisms on serum lipoproteins in patients with viral hepatitis is unknown.

A third unexplored but plausible consequence of TM6SF2 action is influences on viral load in chronic viral hepatitis. Both chronic hepatitis $\mathrm{C}$ and $\mathrm{B}$ ( $\mathrm{CHC}$ and $\mathrm{CHB})$ are associated with abnormalities in lipid metabolism $\left({ }^{21,22,23}\right)$, lipids play important roles in various aspects of the life cycle of the viruses $\left({ }^{24,25}\right)$, and we have shown that a variable fraction of $\mathrm{HCV}$ in serum is associated with triglyceride-rich lipoproteins (TRLs) called lipoviral particles that exhibit increased infectivity $\left({ }^{26}\right)$. Thus, it could be hypothesized that gene variants that modulate hepatic lipid metabolism and TG export could impact on viral load.

Based on these considerations, the aims of the present study were to clarify the influence of the TM6SF2 rs58542926 variant on the metabolic and histological features of liver diseases, comparing viral hepatitis to NAFLD, to explore effects on viral load and thirdly to investigate the impact of carriage of the $\mathrm{T}$ allele on the expression of hepatic TM6SF 2 and genes modulating liver lipid accumulation. 


\section{Methods}

\section{Patient cohort}

The study comprised 3,260 consecutive subjects from the International Liver Disease Genetics Consortium (ILDGC) database. Details of the cohort and inclusion criteria have been reported $\left({ }^{27}\right)$. Briefly, for those with $\mathrm{CHC}$, patients who had a liver biopsy with scoring for fibrosis stage and disease activity before anti-viral treatment were included. Patients were excluded if they had evidence of other liver diseases by standard tests. In addition to the 2,023 with $\mathrm{CHC}$, the cohort comprised 507 patients with CHB, 502 with NAFLD and 228 healthy controls. Only one of the patients with CHC was included in a recent report on TM6SF2 $\left({ }^{15}\right)$. The healthy Caucasian European control group was enrolled from Westmead hospital, Sydney, and reported no history of chronic liver disease. Details of the selection criteria for control subjects are presented in supplementary methods.

Ethics approval was obtained from the Human Research Ethics Committees of the Sydney West Local Health District and the University of Sydney. All other sites had ethics approval from their respective ethics committees. Written informed consent for genetic testing was obtained from all participants.

\section{Clinical and laboratory assessment}

A detailed description of the baseline data collected at the time of liver biopsy and methods to estimate the duration of infection is provided in supplementary methods. Data included demographic parameters, alcohol intake, and routine laboratory variables including metabolic parameters. 


\section{Genotyping}

Genotyping for TM6SF2 rs58542926 and PNPLA3 rs738409 was contracted to the Australian Genome Research Facility (AGRF; QLD, Australia). Samples were genotyped using the Sequenom MassARRAY system and iPLEX Gold chemistry. Further genotyping for both SNPs ( $\mathrm{n}=1150)$ was undertaken using the TaqMan SNP genotyping allelic discrimination method (Applied Biosystems, Foster City, CA, USA). All genotyping was blinded to clinical variables.

\section{Liver Histopathology}

Liver histopathology was scored according to METAVIR $\left({ }^{28}\right)$. Fibrosis was staged from F0 (no fibrosis) to F4 (cirrhosis). Necroinflammation (A) was graded as A0 (absent), A1 (mild), A2 (moderate), or A3 (severe). For NAFLD, the Kleiner classification was used $\left({ }^{29}\right)$.According to the classification of Bedossa et al., $\left({ }^{30}\right)$ histological features of steatohepatitis in $\mathrm{CHC}$ was also assessed in a subset of 112 patients infected with genotype 1. The inter-observer agreement between pathologists was studied previously and was good $(\kappa=77.5)$ for METAVIR staging using $\kappa$ statistics $\left({ }^{31}\right)$. Histopathology methods are detailed in the supplementary methods.

\section{Determination of inflammatory markers and mRNA levels of genes regulating lipid metabolism}

Ninety well characterized patients with $\mathrm{CHC}$ underwent liver biopsy at Westmead Hospital, their details have been described previously $\left({ }^{32}\right)$. We assessed the relationship between rs58542926 genotype and the expression of TM6SF2, acyl-coenzyme A oxidase 1 (ACOX1) and microsomal triglyceride transfer protein $(M T T P)$. The expression of sterol regulatory element binding protein-1c $(S R E B P-1 c)$ and fatty acid synthase $(F A S)$ was measured previously $\left.{ }^{32}\right)$. The

detailed methods have been described previously $\left({ }^{32}\right)$ and summarized in the supplementary 
methods. Serum TNF- $\alpha$, soluble tumor necrosis factor receptor 2 (sTNFR2) and IL-6 in these patients were measured by sandwich enzyme-linked immunosorbent assay (ELISA).

\section{Statistical Analysis}

Statistical methods are detailed in the supplementary methods. All tests were two-tailed and $\mathrm{p}$ values $<0.05$ were considered significant. 


\section{Results}

\section{Patient characteristics}

The study comprised 3,260 consecutive subjects including 2,023 with CHC, 507 with CHB, 502 with NAFLD and 228 healthy controls. The clinical, anthropometric, biochemical and metabolic characteristic of the patients in the $\mathrm{CHC}$ cohort are presented in supplementary table 1. The genotype distribution of TM6SF2 rs58542926 and PNPLA3 rs738409 was in HardyWeinberg equilibrium supplementary table 2.

\section{Association between the TM6SF2 variant and viral, clinical and metabolic characteristics in $\mathrm{CHC}$}

The minor allele frequency (MAF) of TM6SF2 rs58542926 was 0.06, similar to that of the 228 Caucasian self-reported 'healthy' controls (MAF 0.07) ( $\mathrm{p}=0.7$ for trend) supplementary table 1. There was no significant difference in the rs58542926 genotype distribution according to patient country of origin (Australia, UK, Germany and Italy) ( $\mathrm{p}=0.1$ for trend).

Patients with the TM6SF2 rs58542926 TT genotype had significantly lower HCV viral load compared to subjects with $\mathrm{CT}$ or $\mathrm{CC}(\mathrm{p}=0.007)$, after Bonferroni correction for multiple comparisons (Table 1). In another analysis, mean HCV viral load was lower in subjects with CT/TT compared to those with CC (5.74 \pm 0.73 vs. 5.59 $\pm 0.0 .76 ; \mathrm{p}=0.02)$. To adjust for the effect of confounding factors, TM6SF2 rs58542926 TT genotype still associated inversely with HCVRNA levels in a multivariate model adjusting for age, gender, HCV-genotype, BMI, HOMA-IR, lipid profile and PNPAL3 genotype (adjusted estimate, -0.144; SE, 0.082; p=0.001). The distribution of rs58542926 genotype was not different according to HCV genotype.

As regards the metabolic profile, subjects with the $T T$ genotype had significantly lower total cholesterol, LDL-C and TG, while paradoxically having significantly higher BMI and a 
trend to higher HOMA-IR. The median of ALT, AST, GGT and ALP levels, and platelet counts were not significantly different between rs58542926 genotypes (Table 1). Similar results were observed when comparing CT/TT vs. CC (data not shown). For the purposes of comparison, the association of PNPLA3 rs738409 with the same variables was investigated. Apart from the fact that subjects with rs738409 GG genotype were significantly younger and had lower fasting blood glucose level, compared to subjects with $\mathrm{CG}$ and $\mathrm{CC}$, no other significant associations were observed (Supplementary Table 3).

\section{Association between the TM6SF2 variant and histological features in the cohort with CHC}

We next assessed in the CHC cohort, the association between TM6SF2 rs58542926 and histological features including steatosis, features of steatohepatitis (in 112 subjects), inflammation and fibrosis. The distribution of TM6SF2 rs58542926 genotypes according to histological features (steatosis grade, inflammation, and fibrosis) are depicted in Supplementary Figure 1. The rs58542926 $\mathrm{T}$ allele had a modest association with the degree of steatosis in a multivariate model adjusting for age, gender, HCV-genotype, BMI, HOMA-IR and PNPAL3 genotype (adjusted estimate, 0.073; SE, 0.002; p=0.01) (Supplementary Table 4). When stratifying the cohort according to HCV genotype (genotype 3 vs. non-3) based on the direct

effect of genotype 3 on steatosis $\left({ }^{33}\right)$, the rs58542926 was associated with steatosis only in nonHCV-genotype 3 patients (adjusted estimate, 0.112, SE, 0.006; $\mathrm{p}=0.04$ ). In contrast, PNPLA3 rs738409 genotype was the strongest predictor of steatosis in both non-genotype 3 (adjusted estimate, 0.199, SE, 0.14; p=0.005) and in genotype 3 patients (adjusted estimate, 0.127, SE, 0.077; p=0.03) (Supplementary Table 4).

We explored the association of rs58542926 with the severity of steatosis by subdividing the cohort according to absent/mild steatosis (S0-S1) compared to moderate/severe steatosis (S2- 
S3). In this analysis, $r 558542926$ was associated with the severity of steatosis (OR: 1.14, 95\%CI: 1.02-1.27, p=0.01), independent of PNPLA3 rs738409 (OR: 4.3, 95\%CI: 1.94-9.52, p <0.0001) and other clinical variables (Table 2).

We then assessed the role of the TM6SF2 variant on hepatic inflammation. In multivariate analysis, neither TM6SF2 rs58542926, nor PNPLA3 rs738409 demonstrated any association with inflammation (Supplementary Table 5). No association was also observed when the cohort was dichotomized into absent/mild (A0-A1) and moderate/severe (A2-A3) inflammation. In sub-analysis of 112 subjects with genotype $1 \mathrm{CHC}$, we investigated the possible association between TM6SF2 rs58542926 and features of 'steatohepatitis' in CHC according to the Bedossa classification $\left({ }^{30}\right)$ and no association was observed (data not shown). Furthermore, no difference was noted in serum inflammatory markers (TNF- $\alpha$, sTNFR2 and IL-6) or liver enzymes (ALT or AST; as indices of liver injury) according to rs58542926 genotype.

Lastly, in the cross sectional analysis, we examined the impact of TM6SF2 rs58542926 genotype on fibrosis. The rs58542926 SNP was marginally associated with fibrosis stage in univariate analysis, but not in multivariate linear regression analysis controlling for age, gender, steatosis, HOMA-IR, BMI, HCV genotype, alcohol intake and PNPLA3 genotype (adjusted estimate, 0.071, SE, 0.003; $\mathrm{p}=0.06$ ) (Supplementary Table 5). The adjusted OR for having significant fibrosis $(\geq \mathrm{F} 2)$ was 1.39 (95\% CI: $1.04-1.87, \mathrm{p}=0.02)$ for the $\mathrm{T}$ allele, and with cirrhosis $(>\mathrm{F} 4)$ was 1.82 (95\% CI: 1.01-3.28, $\mathrm{p}=0.04)$ (Table 2).

\section{Association between the TM6SF2 variant and fibrosis progression}

Given the inherent biases in cross sectional analyses, the lack of impact of TM6SF2 rs58542926 genotype on hepatic inflammation, the principal driver of liver fibrosis, and the modest impact on fibrosis stage (absent in multivariate linear regression and present by 
multivariate logistic regression), we undertook further analysis in 1,174 patients with CHC and a known duration of infection, allowing us to assess the relationship with fibrosis progression. The baseline characteristics of the cohort were similar among subjects included and not included in the fibrosis progression sub-analysis (Supplementary Table 6). The OR of rs58542926 for having fast FPR was 1.31 (95\% CI: 1.08-2.97, p=0.03), while after adjustment, the T allele was not independently associated with FPR $(\mathrm{p}=0.5)$ (Supplementary Table 7).

For further confirmation, we assessed fibrosis progression using Cox-proportional hazards to address the concern that FPR may not be constant over time $\left({ }^{34}\right)$. rs58542926 was not associated with an increased hazard of progression to significant fibrosis ( $\geq$ F2) in univariate or multivariate models (hazards ratio: 1.43, 95\% CI: 0.64-3.21, p=0.3), including when adopting other genetic models. In contrast, rs738409 in PNPLA3 showed a trend for significance (hazards ratio: $1.34,95 \% \mathrm{CI}: 0.97-1.84, \mathrm{p}=0.06$ ), but again not in a multivariate model (Figure 1). In another model, rs58542926 was not associated with an increased hazard of progression to cirrhosis (F4) (hazards ratio: 1.16, 95\% CI: 0.16-8.39, p=0.8), supplementary figure 2.

There was no evidence of interaction between rs58542926 and rs738409 on any histological features (steatosis, inflammation and fibrosis) (data not shown).

In sum, the TM6SF2 rs58542926 T allele was associated with a modest risk of hepatic steatosis in $\mathrm{CHC}$, being more profound in subjects with non-genotype 3 infection. There was no effect of the T allele on inflammation, while an effect on fibrosis stage was at best, modest, with no effect on fibrosis progression. The effect of the TM6SF2 rs58542926 $\mathrm{T}$ allele was independent of PNPLA3 738409. 


\section{Relationships between $r 55842926$ genotype and hepatic gene expression}

There was a significant relationship between carriage of the rs58542926 $T$ allele and lower TM6SF2 mRNA expression ( $\mathrm{p}=0.001$, Figure 2 B). Expression of SREBPIc, FAS and ACOX1 mRNA, as markers of lipid synthesis and oxidation, were not significantly different according to $r s 58542926$ genotype. As expected however, serum TG was significantly lower in subjects with the $r s 58542926 \mathrm{CT} / \mathrm{TT}$ than CC genotype (Figure 2 C). The expression of MTTP, a key protein involved in TG packaging and export was significantly lower in liver from subjects with the $\mathrm{T}$ allele (Figure 2C). These results suggest that in $\mathrm{CHC}$, carriers of the $\mathrm{T}$ allele for rs58542926 have reduced TM6SF2 and MTTP expression, concomitant with reduced serum TG levels.

\section{Other chronic liver diseases}

\section{Chronic hepatitis B}

The characteristics of 507 Chinese patients with CHB are summarized in Supplementary Table 8. The TM6SF2 rs58542926 MAF of 0.07 was similar to that observed in Caucasian populations, and in other Han Chinese population cohorts $\left({ }^{13}\right)$ (Supplementary Table 1). The distribution of TM6SF2 rs58542926 genotypes according to histological features (steatosis grade, inflammation, and fibrosis) are depicted in Supplementary Figure 3. The rs58542926 T allele was associated with steatosis (adjusted estimate, 0.094; SE, 0.003; $\mathrm{p}=0.03$ ), but, in particular, with presence of any steatosis ( $\mathrm{S} 0$ versus $\mathrm{S} \geq 1)(\mathrm{OR}=2.57$, 95\% CI: 1.36-4.86, $\mathrm{P}=0.003)$ rather than with severe steatosis (S0-S1 versus $\mathrm{S} 2-\mathrm{S} 3)(\mathrm{OR}=1.49,95 \% \mathrm{CI}$ : $0.83-2.67$, $\mathrm{P}=0.1$ ) (Table 3). No association was observed between rs58542926 genotype and inflammation or fibrosis (Supplementary Table 9). Notably, only classic risk factors such as 
age and gender demonstrated an independent association with fibrosis (Supplementary Table 10), and PNPLA3 rs738409 showed no association with either inflammation or fibrosis.

Again, as noted in other liver diseases cohorts, subjects with the $\mathrm{T}$ allele had lower serum TG and LDL-C, but this was significant only for the latter $(\mathrm{p}=0.008)$. Interestingly, in contrast to what was observed in $\mathrm{CHC}$, patients with $\mathrm{CHB}$ had higher HBV-DNA levels $(\mathrm{p}=0.04)$ (Supplementary Table 11). The association with HBV-DNA levels remained significant after adjusting for age, gender, BMI, presence of diabetes, lipid profile and PNPAL3 genotype (adjusted estimate, 0.103; SE, 0.036; $\mathrm{p}=0.03$ ). For PNPLA3 rs 738409 , apart from higher serum HDL-C in subjects with the GG genotype, no other significant associations were demonstrated (Supplementary Table 12).

\section{NAFLD}

The characteristics of 502 Caucasian patients with NAFLD are summarized in Supplementary Table 13. The MAF for $r 558542926$ in the NAFLD cohort was 0.12 with an increase in $\mathrm{T}$ allele frequency compared to healthy controls ( $X^{2}$ for trend, $\mathrm{P}=0.01$; Supplementary Table 1). The distribution of TM6SF2 rs58542926 genotypes according to histological features (steatosis grade, inflammation, and fibrosis) are depicted in Supplementary Figure 4. $r s 58542926$ was associated with the risk of fatty liver (adjusted estimate, 0.111; SE, 0.097; $\mathrm{p}=0.02$ ); this was independent of age, gender, BMI, presence of diabetes, hypertension, and PNPLA3 rs738409 genotype (Supplementary Table 14).

rs58542926 demonstrated an independent association with stage of fibrosis (adjusted estimate, 0.189; SE, 0.117; $p=0.003$ ) and with risk of significant fibrosis ( $\geq$ F2) in multiple logistic regression analysis (OR:1.62, 95\% CI: 1.03.-2.52, p=0.03) (Table 4). Nevertheless, analysis of the relationship between rs58542926 and steatohepatitis did not demonstrate a 
significant association $(\mathrm{p}=0.6)$ (Supplementary Table 14). Likewise, we observed a lack of association between the gene variant and the NAS score (data not shown). There was no interaction between PNPLA3 rs738409 and TM6SF2 rs58542926 variants on any histological features (steatosis, steatohepatitis or fibrosis) in NAFLD.

As expected, subjects with the rs58542926 TT genotype had lower serum TG and ALP levels, while no association was demonstrated with either serum ALT or AST values or with other metabolic parameters (Supplementary Table 15). As a control, the rs738409 PNPLA3 GG genotype demonstrated significantly higher ALT, AST and GGT (Supplementary Table 16), consistent with its independent association with steatohepatitis (adjusted estimate, 0.103; SE, 0.048; $\mathrm{p}=0.03$ ), but no association was observed with the serum lipid profile. In a joint analysis of all cohorts (HCV, HBV, NAFLD), rs58542926 genotype showed no association with ALT, AST or GGT. 


\section{Discussion}

We clarified the influence of the TM6SF2 rs58542926 variant on the metabolic and histological features of viral hepatitis as compared to that in NAFLD. The most noteworthy finding was that the E167K substitution had a consistent effect on the serum metabolic profile and the predisposition to hepatic steatosis, rather than to hepatic inflammation and fibrosis across all three diseases. This effect was independent and not additive to the influence of the PNPLA3 rs738409 C/G (I148M) polymorphism. Consistent with a crucial role for lipids in hepatitis virus replication, a novel observation was that the TM6SF2 $\mathrm{T}$ allele has significant and opposite associations with HCV-RNA and HBV-DNA levels. Mechanistically, we show in a subset of patients, that alteration in MTTP expression in the liver of carriers with the $\mathrm{T}$ allele, might contribute to the hepatic and serum metabolic abnormalities observed.

Across all analyses and disease cohorts, the TM6SF2 $\mathrm{T}$ allele conferred a modest risk for hepatic steatosis with estimates varying from $(0.111,0.073$ and 0.094$)$ in NAFLD, CHC and CHB, respectively, which is less than the risk for PNPLA3 rs738409 (0.168, 0.159 and 0.119 respectively), and for established risk factors such as T2DM, BMI and HCV genotype 3. Likewise, carriage of the $\mathrm{T}$ allele was associated with lower serum lipoproteins in NAFLD, and as we demonstrate for the first time, in chronic viral hepatitis. To gain insights on the function of TM6SF2 in patients with liver disease, we investigated the correlation of rs58542926 genotype with hepatic expression of TM6SF2. The rs58542926 T allele was associated with lower hepatic TM6SF2 gene expression, a finding consistent with that observed by gene expression and eQTL analysis of incidental liver biopsies from 206 patients undergoing aortic valve surgery $\left({ }^{18}\right)$. In that study, patients were segregated according to $r s 10401969$ rather than the rs58542926 genotype. However, rs 10401969 is in strong linkage disequilibrium (LD) with the non synonymous variant $r s 58542926(r 2>0.99)$ identified by the genome-wide exome association 
study $\left({ }^{18}\right)$. This is also consistent with another recent study demonstrating that the rs58542926 T allele is associated with decreased gene and protein expression in liver of NAFLD patients $\left({ }^{12}\right)$.

Of interest, in the CHC sub-cohort, we show that MTTP expression, required for VLDL assembly and maturation was reduced with carriage of the T allele, while the expression of genes involved in lipid synthesis and oxidation (SREBPIc, FAS and Acoxl mRNA) were unaffected.

This human data is consistent with, and adds to recent observations from $\operatorname{Tm} 6 s f 2$ overexpression and knockdown in mice and in vitro studies demonstrating that TM6SF2 is a key modulator of TG-rich lipoproteins and apolipoprotein B (apoB) $\left({ }^{9,18}\right)$. In humans, TG is delivered to adipose tissues by intestinally-derived chylomicrons and by hepatic VLDL synthesis $\left({ }^{35}\right)$. Notably, both liver and intestine express the highest levels of TM6SF2 $\left({ }^{9,18}\right)$. MTTP is absolutely required for VLDL assembly and maturation $\left({ }^{36}\right)$, catalyzing the transport of triglyceride, cholesteryl ester and phospholipids to the nascent apoB molecule, that is required for the assembly and secretion of VLDL and low-density lipoproteins (LDLs) $\left({ }^{37}\right)$. MTTP is found in abundance on the luminal side of the ER and in the liver, intestine, and heart $\left({ }^{38}\right)$, and thus it is of interest that a recent study using confocal microscopy observed localization of green fluorescent protein-tagged TM6SF2 to the ER of 2 human hepatoma cell lines (Huh7 and HepG2) $\left({ }^{18}\right)$. In that study, knockdown of TM6SF2 in both cell lines had no effect on the expression of $A P O B$ or $M T T P$, however whether $M T T P$ expression is altered based on TM6SF2 genotype was not tested.

Of particular interest is the significant and opposite association between the TM6SF2 variant and $\mathrm{HCV}$ and $\mathrm{HBV}$ viral load, with the T allele associated with lower HCV-RNA levels but higher HBV-DNA. The association remained significant ( $\mathrm{p}=0.001$ with $\mathrm{CHC}$ and $\mathrm{p}=0.03$ with $\mathrm{CHB}$ ) after controlling for the main confounders. Although our analyses do not allow for a 
mechanistic explanation, it is consistent with previous reports from population based studies and experimental models indicating that HCV-RNA levels correlate positively, while HBV-DNA levels correlate inversely with serum TG $\left({ }^{21-23}\right)$. From a teleological standpoint, the question remains how the E167K substitution in TM6SF2 rs58542926 might favor HBV replication and inhibit HCV. Previous studies have shown that blocking VLDL secretion by inhibiting MTTP with naringenin reduces the export of $\mathrm{HCV}\left({ }^{39}\right)$, while overexpression of seipin, a protein implicated in the maturation and fusion of lipid droplets (LDs) in hepatoma cells subsequently infected with $\mathrm{HCV}$ causes a significant decrease in virion export, accompanied by the appearance of large LDs (experimental steatosis) $\left({ }^{40}\right)$. Thus, our data if confirmed suggests that the functional consequence of the E167K substitution in TM6SF2 by blocking VLDL export might also restrict HCV export and hence viral load.

Although the interaction of the HBV life cycle with lipid metabolism is not well characterized, it has been suggested that lipids play a vital role in HBV replication $\left({ }^{41}\right)$. While acute hepatitis B is associated with transient hypertriglyceridemia $\left({ }^{42}\right), \mathrm{CHB}$ is associated with reduced serum TG and total cholesterol $\left({ }^{43}\right)$. Several studies suggest that the HBV X protein (HBx) induces lipid accumulation in HepG2-HBx stable cells and in HBx-transgenic mice $\left({ }^{44,45}\right.$ ) by activation of lipogenic and fatty acid synthesis genes such as SREBP1 and peroxisome proliferator-activated receptor $\gamma(P P A R \gamma)$, and inhibiting the secretion of apoB. Likewise, in HepG2-HBV-stable cells, virus replication upregulates adipogenic gene expression (CCAAT/enhancer binding protein $\alpha(C / E B P \alpha), P P A R \gamma$, adiponectin, liver $X$ receptor $\alpha(L X R \alpha)$, SREBPIc, and Fas). Further, adiponectin and the PPAR $\gamma$ agonist rosiglitazone stimulate viral replication $\left({ }^{46}\right)$. Thus, promotion of lipid synthesis, and blockage of lipid secretion are important pathways in HBV infection suggesting that hepatic steatosis induced by the E167K substitution could promote HBV replication. However, this hypothesis needs further investigation. 
A recent study has suggested that the prevalence of the $r 558542926 \mathrm{~T}$ allele is reduced in European Caucasians with $\mathrm{CHC}\left({ }^{15}\right)$, a finding we have been unable to replicate in the total cohort with $\mathrm{CHC}$ or in the different European and Australian centers, or in a the cohort of Asians with chronic hepatitis B. The reason for this discrepancy is not clear. Of relevance, whether rs58542926 is involved in HCV or HBV spontaneous clearance requires further investigation. The TM6SF2 rs58542926 MAF of 0.07 in our Chinese patients with CHB was similar to that observed in Caucasian populations, and in other Han Chinese population cohorts $\left({ }^{13}\right)$.

In our three cohorts, we were able to undertake robust analysis to assess the role of the E167K substitution on the more controversial aspects of liver histology, namely inflammation and fibrosis. The TM6SF2 rs58542926 variant had no detectable effect on inflammation in all three chronic liver diseases, whether by histological grade, liver enzymes (ALT or AST) or in a subset with CHC, the levels of serum inflammatory markers (TNF- $\alpha$, IL-6 and sTNFR2). Thus, clearly, if the E167K substitution in TM6SF2 rs58542926 contributes to liver fibrosis, as has been suggested $\left({ }^{10,15}\right)$ it is likely mediated by a process other than hepatic necroinflammation.

To confirm or refute a role for the E167K substitution in TM6SF2 on liver fibrosis, the cohort of nearly 1200 patients with $\mathrm{CHC}$ and a known duration of infection had us uniquely positioned to assess fibrosis progression, compared to all previous reports. In this analysis, rs58542926 genotype had no effect on fibrosis progression assessed using either stage-constant fibrosis progression rates or Cox regression to model the time taken for significant fibrosis $(\geq$ F2) or cirrhosis ( $\geq$ F4) to develop. Likewise, in cross sectional analyses of the total cohort with CHC ( $\mathrm{n}=2023)$, the rs58542926 T allele was not associated with fibrosis stage in adjusted multivariate linear regression analysis, while it only showed an association with $\geq \mathrm{F} 2$ fibrosis (OR: 1.39) and with cirrhosis (OR: 1.82) by logistic regression, consistent with a recent report $\left({ }^{15}\right)$. In CHB, likewise, we demonstrated no significant association of the rs58542926 variant 
with fibrosis stage, significant fibrosis (F2-F4), severe fibrosis (F3-F4) or with cirrhosis. In cross sectional analysis of the NAFLD cohort, as with previous reports $\left({ }^{10,11}\right)$, we confirmed an association of the rs58542926 variant with more severe fibrosis stage and with significant fibrosis. The adjusted estimate and $\mathrm{OR}$ for $r s 58542926$ ( $\beta=0.136$ and $\mathrm{OR}=1.62$, respectively) was lower than these values for either PNPLA3 $r s 738409$ ( $\beta=0.1819$ and $\mathrm{OR}=1.98$, respectively) or classic clinical risk variables such as age, diabetes and hypertension.

Thus, in sum, our analyses indicates that the E167K substitution in TM6SF2 rs58542926 has marginal if any effect on fibrosis in chronic viral hepatitis, and no effect on inflammation or fibrosis progression. In NAFLD, perhaps because steatosis occurs in the context of systemic metabolic dysregulation and oxidant stress from intra-hepatic toxic lipid species such as unesterified free fatty acids and ceramides $\left({ }^{47}\right)$, the TM6SF2 rs58542926 variant more consistently amplifies and contributes to fibrosis. It should be noted, that the present analysis is unable to dissect whether the effect of the TM6SF2 variant on fibrosis is direct or indirect through its association with metabolic derangements.

Unfortunately, data on viral clearance was not available in order to investigate the clinical relevance of our observations regarding TM6SF2 rs58542926 with HCV and HBV viral load. Further, it should be acknowledged that population stratification was not assessed in detail. However, all participants with CHC and NAFLD were of Caucasian ancestry and all CHB patients were Chinese. It should be noted that population structure is more critical when studying rare variants $\left({ }^{48}\right.$ ), while $r s 58542926$ does not fit into this category (MAF=0.07), and we did not observe any difference in MAF between the different centres.

In conclusion, this is the first study to compare the diverse impacts of the rs58542926 E167K variant in TM6SF2 on the metabolic and histological features of metabolic and viral liver 
disease. We demonstrate that the E167K substitution in TM6SF2 is associated with altered serum lipoproteins and susceptibility to hepatic steatosis in viral and non viral chronic liver disease, and with reduced hepatic MTTP expression in the HCV cohort. Though, the TM6SF2 rs58542926 minor allele influenced fibrosis in NAFLD, it had no discernable effects on liver inflammation, and perhaps only a minor influence on hepatic fibrosis, in chronic viral hepatitis. Importantly, the variant is associated with viral load, in opposing directions in $\mathrm{CHC}$ as compared to $\mathrm{CHB}$, likely modulated by the differential effects of hepatic lipid metabolism and export, on the HBV and HCV virus life cycle. 


\section{Collaborators:}

\section{The International Liver Disease Genetics Consortium (ILDGC)}

Rose White ${ }^{1}$, Angela Rojas ${ }^{15}$, Rocio Gallego-Duran ${ }^{15}$, Margaret Bassendine ${ }^{17}$, Vincent W.S. Wong $^{5}$, Chiara Rosso ${ }^{9}$, Lavinia Mezzabotta ${ }^{9}$, Reynold Leung ${ }^{1,20}$, Barbara Malik ${ }^{3}$, Gail Matthews $^{7}$, Tanya Applegate ${ }^{25}$, Jason Grebely ${ }^{7}$, Vincenzo Fragomeli ${ }^{15}$, Julie R. Jonsson ${ }^{22}$, Rosanna Santaro ${ }^{2}$ 


\section{Figures legends:}

Figure 1: Univariate Cox regression analysis of TM6SF2 rs58542926 and PNPLA3 rs738409 genotype on the cumulative probability of progression to moderate/severe $(\geq F 2)$ fibrosis in 1174 patients with a known duration of HCV infection. Similar results was observed in multivariate Cox regression analysis after adjusting for covariates (age, gender, BMI, duration of the infection, HCV genotype, inflammation progression, and basal ALT, AST, GGT, platelets, bilirubin and alkaline phosphatase). Data of TM6SF2 rs58542926 and PNPLA3 rs738409 genotype are shown in dominant and additive models, respectively. No difference was observed in other genetic models.

Figure 2: A) Relationship between $r s 58542926$ genotype and serum triglyceride level in 1059 patients with CHC. In human liver samples from patients with CHC: B) Relationship between rs58542926 genotype and TM6SF2 mRNA levels C) Relationship between rs58542926 genotype and the expression of sterol regulatory element binding protein-1c (SREBP-1c), fatty acid synthase $(F A S)$, acyl-coenzyme A oxidase $1(A C O X 1)$ and microsomal triglyceride transfer protein (MTTP). 


\section{REFERENCES}

${ }^{1} \mathrm{Ng}$ M, Fleming T, Robinson M, Thomson B, Graetz N, Margono C, et al. Global, regional, and national prevalence of overweight and obesity in children and adults during 1980-2013: a systematic analysis for the Global Burden of Disease Study 2013. Lancet 2014;384(9945):76681.

${ }^{2}$ Lumeng CN, Saltiel AR. Inflammatory links between obesity and metabolic disease. J Clin Invest 2011, 121(6):2111-7.

${ }^{3}$ Adams LA, Lymp JF, St Sauver J, Sanderson SO, Lindor KD, Feldstein A, et al. The natural history of nonalcoholic fatty liver disease: a population-based cohort study. Gastroenterology. 2005;129(1):113-21.

${ }^{4}$ Fabbrini E, Magkos F, Mohammed BS, Pietka T, Abumrad NA, Patterson BW, et al. Intrahepatic fat, not visceral fat, is linked with metabolic complications of obesity. Proc Natl Acad Sci U S A. 2009;106(36):15430-5.

${ }^{5}$ Gordon A, McLean CA, Pedersen JS, Bailey MJ, Roberts SK. Hepatic steatosis in chronic hepatitis B and C: predictors, distribution, and effect on fibrosis. J Hepatol 2005, 43:38-44.

${ }^{6}$ Romeo S, Kozlitina J, Xing C, Pertsemlidis A, Cox D, Pennacchio LA, et al. Genetic variation in PNPLA3 confers susceptibility to nonalcoholic fatty liver disease. Nat Genet. 2008;40(12):1461-5.

7 Speliotes EK, Yerges-Armstrong LM, Wu J, Hernaez R, Kim LJ, Palmer CD, et al.Genomewide association analysis identifies variants associated with nonalcoholic fatty liver disease that have distinct effects on metabolic traits. PLoS Genet. 2011;7(3):e1001324. 
${ }^{8}$ Valenti L, Al-Serri A, Daly AK, Galmozzi E, Rametta R, Dongiovanni P, et al. Homozygosity for the patatin-like phospholipase-3/adiponutrin I148M polymorphism influences liver fibrosis in patients with nonalcoholic fatty liver disease. Hepatology. 2010;51(4):1209-17

${ }^{9}$ Kozlitina J, Smagris E, Stender S, Nordestgaard BG, Zhou HH, Tybjærg-Hansen A, et al. Exome-wide association study identifies a TM6SF2 variant that confers susceptibility to nonalcoholic fatty liver disease. Nat Genet. 2014;46(4):352-6.

10 Liu YL, Reeves HL, Burt AD, Tiniakos D, McPherson S, Leathart JB, et al. TM6SF2 rs58542926 influences hepatic fibrosis progression in patients with non-alcoholic fatty liver disease. Nat Commun. $2014 ; 5: 4309$.

11 Dongiovanni P, Petta S, Maglio C, Fracanzani AL, Pipitone R, Mozzi E, et al. Transmembrane 6 superfamily member 2 gene variant disentangles nonalcoholic steatohepatitis from cardiovascular disease. Hepatology 2015;61:506-514.

12 Sookoian S, Castaño GO, Scian R, Mallardi P, Fernández Gianotti T, Burgueño AL, et al. Genetic variation in transmembrane 6 superfamily member 2 and the risk of nonalcoholic fatty liver disease and histological disease severity. Hepatology. 2015;61(2):515-25.

13 Wong VW, Wong GL, Tse CH, Chan HL. Prevalence of the TM6SF2 variant and nonalcoholic fatty liver disease in Chinese. J Hepatol. 2014 ;61(3):708-9.

${ }^{14}$ Arslanow A, Stokes CS, Weber SN, Grünhage F, Lammert F, Krawczyk M. The common PNPLA3 variant p.I148M is associated with liver fat contents as quantified by controlled attenuation parameter (CAP). Liver Int. 2015 Aug 12.

${ }_{15}$ Milano M, Aghemo A, Mancina RM, Fischer J, Dongiovanni P, De Nicola S, et al. Transmembrane 6 superfamily member 2 gene E167K variant impacts on steatosis and liver damage in chronic hepatitis C patients. Hepatology 2015, 62(1):111-7. 
16 Petta S, Maida M, Grimaudo S, Pipitone RM, Macaluso FS, Cabibi D, et al.TM6SF2 rs58542926 is not associated with steatosis and fibrosis in large cohort of patients with genotype 1 chronic hepatitis C. Liver Int. 2015 Jul 17.

${ }^{17}$ Pirola CJ, Sookoian S. The dual and opposite role of the TM6SF2-rs58542926 variant in protecting against cardiovascular disease and conferring risk for nonalcoholic fatty liver: A metaanalysis. Hepatology. 2015;62(6):1742-56.

18 Mahdessian H, Taxiarchis A, Popov S, Silveira A, Franco-Cereceda A, Hamsten A, et al. TM6SF2 is a regulator of liver fat metabolism influencing triglyceride secretion and hepatic lipid droplet content. Proc Natl Acad Sci U S A 2014;111:8913-8918.

19 Steenbergen RH, Joyce MA, Thomas BS, Jones D, Law J, Russell R, et al. Human serum leads to differentiation of human hepatoma cells, restoration of very-low-density lipoprotein secretion, and a 1000-fold increase in $\mathrm{HCV}$ Japanese fulminant hepatitis type 1 titers. Hepatology. 2013;58(6):1907-17.

${ }^{20}$ Meex SJ, Andreo U, Sparks JD, Fisher EA. Huh-7 or HepG2 cells: which is the better model for studying human apolipoprotein-B100 assembly and secretion? J Lipid Res. 2011;52(1):152-8. ${ }^{21}$ Hsu CS, Liu CH, Liu CJ, Wang CC, Chen CL, Lai MY, et al. Association of lipid profiles with hepatitis $\mathrm{C}$ viral load in chronic hepatitis $\mathrm{C}$ patients with genotype 1 or 2 infection. Am J Gastroenterol. 2009;104(3):598-604.

${ }^{22}$ Khattab MA, Eslam M, Aly MM, Shatat M, Mousa YI, Abd-Aalhalim H, et al. Serum lipids and chronic hepatitis C genotype 4: interaction and significance. Ann Hepatol. 2012;11(1):37-46. ${ }^{23}$ Chiang CH, Yang HI, Jen CL, Lu SN, Wang LY, You SL, et al. Association between obesity, hypertriglyceridemia and low hepatitis B viral load. Int J Obes (Lond). 2013;37(3):410-5. 
${ }^{24}$ Ye J. liance of host cholesterol metabolic pathways for the life cycle of hepatitis $\mathrm{C}$ virus. PLoS Pathog. 2007;3(8):e108.

${ }^{25}$ Xie N, Huang K, Zhang T, Lei Y, Liu R, Wang K, et al. Comprehensive proteomic analysis of host cell lipid rafts modified by HBV infection. J Proteomics. 2012;75(3):725-39.

${ }^{26}$ Bridge SH, Sheridan DA, Felmlee DJ, Nielsen SU, Thomas HC, Taylor-Robinson SD, et al. Insulin resistance and low-density apolipoprotein B-associated lipoviral particles in hepatitis C virus genotype 1 infection. Gut. 2011;60(5):680-7.

${ }^{27}$ Eslam M, Hashem AM, Leung R, Romero-Gomez M, Berg T, Dore GJ, et al. Interferon- $\lambda$ rs12979860 genotype and liver fibrosis in viral and non-viral chronic liver disease. Nat Commun $2015,6: 6422$.

${ }^{28}$ Bedossa P, Poynard T. An algorithm for the grading of activity in chronic hepatitis C. The METAVIR Cooperative Study Group. Hepatology 1996, 24:289.

${ }^{29}$ Kleiner DE, Brunt EM, Van Natta M, Behling C, Contos MJ, Cummings OW, et al. Design and validation of a histological scoring system for nonalcoholic fatty liver disease. Hepatology 2005, 41:1313-1321.

${ }^{30}$ Bedossa P, Moucari R, Chelbi E, Asselah T, Paradis V, Vidaud M, et al. Evidence for a role of nonalcoholic steatohepatitis in hepatitis C: a prospective study. Hepatology 2007, 46: 380-7.

${ }^{31}$ Eslam M, Leung R, Romero-Gomez M, Mangia A, Irving WL, Sheridan D, et al. IFNL3 polymorphisms predict response to therapy in chronic hepatitis C genotype $2 / 3$ infection. J Hepatol 2014, 61(2):235-41.

${ }^{32}$ van der Poorten D, Shahidi M, Tay E, Sesha J, Tran K, McLeod D, Milliken JS, et al. Hepatitis C virus induces the cannabinoid receptor 1. PLoS One. 2010, 5(9). pii: e12841. 
${ }^{33}$ Hui JM, Kench J, Farrell GC, Lin R, Samarasinghe D, Liddle C, et al. Genotype-specific mechanisms for hepatic steatosis in chronic hepatitis C infection. J Gastroenterol Hepatol 2002, 17: $873-881$

${ }^{34}$ Poynard T, Ratziu V, Charlotte F, Goodman Z, McHutchison J, Albrecht J. Rates and risk factors of liver fibrosis progression in patients with chronic hepatitis C. J Hepatol 2001, 34: 7309.

${ }^{35}$ Lee RG, Shah R, Sawyer JK, Hamilton RL, Parks JS, Rudel LL. ACAT2 contributes cholesteryl esters to newly secreted VLDL, whereas LCAT adds cholesteryl ester to LDL in mice. J Lipid Res 2005, 46:1205-1212.

${ }^{36}$ Jamil H, Chu CH, Dickson JK Jr, Chen Y, Yan M, Biller SA, et al. Evidence that microsomal triglyceride transfer protein is limiting in the production of apolipoprotein B-containing lipoproteins in hepatic cells. J Lipid Res 1998, 39:1448-1454.

${ }^{37}$ Fisher EA, Ginsberg HN. Complexity in the secretory pathway: the assembly and secretion of apolipoprotein B-containing lipoproteins. J Biol Chem 2002, 277:17377-17380.

${ }^{38}$ Wetterau JR, Zilversmit DB. Localization of intracellular triacylglycerol and cholesteryl ester transfer activity in rat tissues. Biochim Biophys Acta 1986, 875:610-617.

${ }^{39}$ Nahmias Y, Goldwasser J, Casali M, van Poll D, Wakita T, Chung RT, et al. Apolipoprotein B-dependent hepatitis $\mathrm{C}$ virus secretion is inhibited by the grapefruit flavonoid naringenin. Hepatology 2008, 47(5):1437-45.

${ }^{40}$ Clément S, Fauvelle C, Branche E, Kaddai V, Conzelmann S, Boldanova T, et al. Role of seipin in lipid droplet morphology and hepatitis C virus life cycle. J Gen Virol 2013, 94(Pt 10):2208-14. 
${ }^{41}$ Geier A. Hepatitis B virus: the "metabolovirus" highjacks cholesterol and bile acid metabolism. Hepatology 2014, 60(5):1458-60.

${ }^{42}$ Mordasini RC, Berthold S, Schlumpf E, Riva G. Changes in serum lipids and lipoproteins in acute hepatitis. Schweiz Med Wochenschr 1976, 106(35):1173-82.

${ }^{43}$ Hsu CS, Liu CH, Wang CC, Tseng TC, Liu CJ, Chen CL, et al. Impact of hepatitis B virus infection on metabolic profiles and modifying factors. J Viral Hepat 2012, 19(2):e48-57.

${ }^{44} \mathrm{Kim} \mathrm{KH}$, Shin HJ, Kim K, Choi HM, Rhee SH, Moon HB, et al. Hepatitis B virus X protein induces hepatic steatosis via transcriptional activation of SREBP1 and PPARgamma. Gastroenterology 2007, 132(5):1955-67.

${ }^{45} \mathrm{Na}$ TY, Shin YK, Roh KJ, Kang SA, Hong I, Oh SJ, et al. Liver X receptor mediates hepatitis B virus $\mathrm{X}$ protein-induced lipogenesis in hepatitis B virus-associated hepatocellular carcinoma. Hepatology 2009, 49:1122-1131.

${ }^{46}$ Yoon S, Jung J, Kim T, Park S, Chwae YJ, Shin HJ, et al. Adiponectin, a downstream target gene of peroxisome proliferator-activated receptor $\gamma$, controls hepatitis B virus replication. Virology 2011, 409(2):290-8.

${ }^{47}$ Greco D, Kotronen A, Westerbacka J, Puig O, Arkkila P, Kiviluoto T, et al. Gene expression in human NAFLD. Am J Physiol Gastrointest Liver Physiol 2008, 294(5):G1281-7.

${ }^{48}$ Lee S, Abecasis GR, Boehnke M, Lin X. Rare-variant association analysis: study designs and statistical tests. Am J Hum Genet 2014;95:5-23. 